MIFP-08-29

\title{
Kerr-AdS/CFT Correspondence in Diverse Dimensions
}

\author{
H. Lü ${ }^{\dagger}$, Jianwei Mei ${ }^{\dagger}$ and C.N. Pope ${ }^{\dagger, \ddagger}$ \\ ${ }^{\dagger}$ George and Cynthia Woods Mitchell Institute for Fundamental Physics and Astronomy, \\ Texas A\&M University, College Station, TX 77843, USA \\ ‡DAMTP, Centre for Mathematical Sciences, Cambridge University, \\ Wilberforce Road, Cambridge CB3 OWA, UK
}

\begin{abstract}
$\underline{\text { ABSTRACT }}$
It was proposed recently that the near-horizon states of an extremal four-dimensional Kerr black hole could be identified with a certain chiral conformal field theory whose Virasoro algebra arises as an asymptotic symmetry algebra of the near-horizon Kerr geometry. Supportive evidence for the proposed duality came from the equality of the microscopic entropy of the CFT, calculated by means of the Cardy formula, and the Bekenstein-Hawking entropy of the extremal Kerr black hole. In this paper we examine the proposed Kerr/CFT correspondence in a broader context. In particular, we show that the microscopic entropy and the Bekenstein-Hawking entropy agree also for the extremal Kerr-AdS metric in four dimensions, and also for the extremal Kerr-AdS metrics in dimensions 5, 6 and 7. General formulae for all higher dimensions are also presented.
\end{abstract}




\section{Introduction}

In a recent paper, it was shown that the near-horizon states of an extremal four-dimensional Kerr black hole could be identified with a certain two-dimensional chiral conformal field theory [1]. This CFT arises by considering the asymptotic symmetry generators associated with a class of perturbations around the near-horizon Kerr geometry that obey suitablychosen boundary conditions. The Cardy formula then gives a microscopic formula for the entropy for the CFT, and it was shown that this coincides with the Bekenstein-Hawking entropy of the extremal Kerr black hole. This led the authors of [1] to conjecture that the extreme Kerr black hole is holographically dual to a chiral two-dimensional CFT whose central charge is proportional to the angular momentum of the black hole. (See [2-6] for some earlier related work.)

In this paper, we test the conjectured Kerr/CFT Correspondence for a wider variety of extremal rotating black holes. Firstly, we consider the case of the Kerr-AdS black hole in four dimensions, beginning with the construction of the near-horizon geometry of the extremal Kerr-AdS black hole [7]. We show that again in this generalisation to include a cosmological constant, there is an agreement between the Cardy formula for the CFT and the BekensteinHawking entropy of the extremal black hole. We then turn to the consideration of higherdimensional rotating black holes [8-10], in the extremal limit, beginning with a detailed discussion of the five-dimensional case [11]. In dimensions higher than four, there is the new feature that there is more than one angular momentum, since the black hole can rotate independently in multiple mutually-orthogonal spatial 2-planes. We now find that there is a chiral two-dimensional CFT associated with each of these rotations. The central charge is different for each CFT, but nevertheless, the Cardy formula gives rise to the same microscopic entropy for the states in each CFT, and furthermore, this microscopic entropy matches perfectly with the Bekenstein-Hawking entropy of the extremal rotating black hole. This agreement holds for both Ricci-flat black holes and those with a cosmological constant. Having demonstrated this explicitly for the case of five-dimensional extremal rotating black holes, we then present the analogous results for all higher dimensions. We have explicitly verified these expressions, and hence the conjectured duality, in the cases of six and seven dimensional extremal black holes. Our results include a construction of the metrics describing the near-horizon geometries for extremal Kerr-AdS black holes in all dimensions. 


\section{Four-Dimensional Extremal Kerr-AdS Black Holes}

\subsection{The near-horizon metric}

The metric of the four-dimensional Kerr-AdS black hole, satisfying $R_{\mu \nu}=-3 \ell^{-2} g_{\mu \nu}$, is given by [7]

$$
\begin{aligned}
d s^{2}= & \rho^{2}\left(\frac{d \hat{r}^{2}}{\Delta}+\frac{d \theta^{2}}{\Delta_{\theta}}\right)+\frac{\Delta_{\theta} \sin ^{2} \theta}{\rho^{2}}\left(a d \hat{t}-\frac{\hat{r}^{2}+a^{2}}{\Xi} d \hat{\phi}\right)^{2}-\frac{\Delta}{\rho^{2}}\left(d \hat{t}-\frac{a \sin ^{2} \theta}{\Xi} d \hat{\phi}\right)^{2}, \\
\rho^{2}= & \hat{r}^{2}+a^{2} \cos ^{2} \theta, \quad \Delta=\left(\hat{r}^{2}+a^{2}\right)\left(1+\hat{r}^{2} \ell^{-2}\right)-2 M \hat{r}, \\
& \Delta_{\theta}=1-a^{2} \ell^{-2} \cos ^{2} \theta, \quad \Xi=1-a^{2} \ell^{-2} .
\end{aligned}
$$

The outer horizon is located at $r=r_{+}$, the largest root of $\Delta=0$. Note that in this coordinate system the metric is asymptotic to $\mathrm{AdS}_{4}$ in a rotating frame, with angular velocity $\Omega_{\infty}=-a \ell^{-2}$.

The Hawking temperature, the Bekenstein-Hawking entropy, the angular momentum and the angular velocity of the horizon (as measured in the asymptotically rotating frame) are given by

$$
\begin{aligned}
T_{H} & =\frac{r_{+}^{2}-a^{2}+r_{+}^{2} \ell^{-2}\left(3 r_{+}^{2}+a^{2}\right)}{4 \pi r_{+}\left(r_{+}^{2}+a^{2}\right)}, \quad S_{B H}=\frac{\pi\left(r_{+}^{2}+a^{2}\right)}{\Xi}, \\
J_{\phi} & =\frac{M a}{\Xi^{2}}, \quad \Omega_{\phi}=\frac{a \Xi}{r_{+}^{2}+a^{2}} .
\end{aligned}
$$

The extremal limit is attained when the function $\Delta$ has a double zero at the outer horizon, which we shall denote by $r=r_{0}$, i.e. when $\Delta\left(r_{0}\right)=0$ and $\Delta^{\prime}\left(r_{0}\right)=0$, implying that the Hawking temperature vanishes. In order to avoid expressions explicitly involving roots of the polynomial equation, it is convenient to use these two conditions in order to express the mass parameter $M$ and the rotation parameter $a$ in terms of $r_{0}$ :

$$
M=\frac{r_{0}\left(1+r_{0}^{2} \ell^{-2}\right)^{2}}{1-r_{0}^{2} \ell^{-2}}, \quad a^{2}=\frac{r_{0}^{2}\left(1+3 r_{0}^{2} \ell^{-2}\right)}{1-r_{0}^{2} \ell^{-2}}
$$

Near the horizon of the extremal black hole, the function $\Delta$ takes the form

$$
\Delta=\left(\hat{r}-r_{0}\right)^{2} V+\mathcal{O}\left(\left(\hat{r}-r_{0}\right)^{3}\right), \quad \text { with } \quad V=\frac{1+6 r_{0}^{2} \ell^{-2}-3 r_{0}^{4} \ell^{-4}}{1-r_{0}^{2} \ell^{-2}}
$$

To obtain the near-horizon geometry, we make the coordinate transformations

$$
\hat{r}=r_{0}(1+\lambda y), \quad \hat{\phi}=\phi+\frac{a \Xi}{r_{0}^{2}+a^{2}} \hat{t},
$$

and scale the time $\hat{t}$ by writing

$$
\hat{t}=\frac{r_{0}^{2}+a^{2}}{\lambda r_{0} V} t
$$


Sending $\lambda \rightarrow 0$ then gives the near-horizon metric

$$
\begin{aligned}
d s^{2} & =\frac{\rho_{0}^{2}}{V}\left(-y^{2} d t^{2}+\frac{d y^{2}}{y^{2}}+\frac{V d \theta^{2}}{\Delta_{\theta}}\right)+\frac{\sin ^{2} \theta \Delta_{\theta}}{\rho_{0}^{2}}\left(\frac{2 a r_{0}}{V} y d t+\frac{r_{0}^{2}+a^{2}}{\Xi} d \phi\right)^{2}, \\
\rho_{0}^{2} & =r_{0}^{2}+a^{2} \cos ^{2} \theta .
\end{aligned}
$$

Finally, we can transform the $\mathrm{AdS}_{2}$ Poincaré coordinates $y$ and $t$ to global coordinates $r$ and $\tau$ as follows. $\mathrm{AdS}_{2}$ is described by the hyperboloid $Z^{2}-X^{2}-Y^{2}=-1$ in $\mathbb{R}^{3}$, whose parameterisations in the Poincaré and global coordinate systems are

$$
\begin{aligned}
\text { Poincaré : } & X+Z=y, \quad X-Z=\frac{1}{y}-y t^{2}, \quad Y=y t, \\
\text { Global : } & X=\sqrt{1+r^{2}} \cos \tau, \quad Y=\sqrt{1+r^{2}} \sin \tau, \quad Z=r .
\end{aligned}
$$

From this, we see that

$$
y=r+\sqrt{1+r^{2}} \cos \tau, \quad t=\frac{\sqrt{1+r^{2}} \sin \tau}{r+\sqrt{1+r^{2}} \cos \tau},
$$

and hence we find that

$$
\begin{aligned}
-y^{2} d t^{2}+\frac{d y^{2}}{y^{2}} & =-\left(1+r^{2}\right) d \tau^{2}+\frac{d r^{2}}{1+r^{2}} \\
y d t & =r d \tau+d \gamma
\end{aligned}
$$

where

$$
\gamma \equiv \log \left(\frac{1+\sqrt{1+r^{2}} \sin \tau}{\cos \tau+r \sin \tau}\right)
$$

Thus by sending

$$
\phi \longrightarrow \phi+\frac{2 a r_{0} \Xi \gamma}{\left(r_{0}^{2}+a^{2}\right) V},
$$

the near-horizon metric (2.7) becomes

$$
d s^{2}=\frac{\rho_{0}^{2}}{V}\left(-\left(1+r^{2}\right) d \tau^{2}+\frac{d r^{2}}{1+r^{2}}+\frac{V d \theta^{2}}{\Delta_{\theta}}\right)+\frac{\sin ^{2} \theta \Delta_{\theta}}{\rho_{0}^{2}}\left(\frac{2 a r_{0}}{V} r d \tau+\frac{r_{0}^{2}+a^{2}}{\Xi} d \phi\right)^{2},
$$

In the case of vanishing cosmological constant, the metric becomes the near-horizon geometry of the Kerr solution, analysed in [12].

\subsection{Central charge in the Virasoro algebra}

Here we follow the procedures described in [1], in which the asymptotic symmetry algebra for a certain class of diffeomorphisms of the near-horizon extremal metric is studied. A crucial aspect of these diffeomorphisms is that the deviations $h_{\mu \nu}$ of the background metric are allowed, in the $h_{\tau \tau}$ and $h_{\phi \phi}$ directions, to be of the same order in inverse powers of $r$ as the 
corresponding components in the background metric itself. This is less restrictive than the asymptotically AdS boundary conditions considered in [13], where all metric components are required to be at sub-leading order. We refer the reader to [1] for a detailed discussion of this point. By allowing the more general boundary conditions, it was shown in [1] that a class of diffeomorphisms around the near-horizon extremal Kerr metric gave rise to a chiral Virasoro algebra. We now apply the analogous analysis to the case with a cosmological constant.

The relevant diffeomorphisms are given by the vector fields [1]

$$
\zeta_{\epsilon}=\epsilon(\phi) \frac{\partial}{\partial \phi}-r \epsilon^{\prime}(\phi) \frac{\partial}{\partial r} .
$$

The function $\epsilon(\phi)$ is periodic in the azimuthal angle, and we may consider a mode expansion in terms of diffeomorphisms $\zeta_{(n)}$ in which we take $\epsilon(\phi)=-e^{-\mathrm{in} \phi}$ :

$$
\zeta_{(n)}=-e^{-\mathrm{i} n \phi} \frac{\partial}{\partial \phi}-\mathrm{i} n r e^{-\mathrm{i} n \phi} \frac{\partial}{\partial r} .
$$

The commutator of these vector fields generates the centreless Virasoro algebra,

$$
\mathrm{i}\left[\zeta_{(m)}, \zeta_{(n)}\right]=(m-n) \zeta_{(m+n)}
$$

Following the discussion in [1], charges $Q_{\zeta}$ associated with these diffeomorphisms are defined by integrals over a spatial slice $\partial \Sigma$,

$$
Q_{\zeta}=\frac{1}{8 \pi} \int_{\partial \Sigma} k_{\zeta}
$$

where the 2 -form $k_{\zeta}$ is defined for a perturbation $h_{\mu \nu}$ around the background metric $g_{\mu \nu}$ by

$$
\begin{gathered}
k_{\zeta}[h, g]=\frac{1}{2}\left[\zeta_{\nu} \nabla_{\mu} h-\zeta_{\nu} \nabla_{\sigma} h_{\mu}{ }^{\sigma}+\zeta_{\sigma} \nabla_{\nu} h_{\mu}{ }^{\sigma}+\frac{1}{2} h \nabla_{\nu} \zeta_{\mu}-h_{\nu}{ }^{\sigma} \nabla_{\sigma} \zeta_{\mu}\right. \\
\left.\frac{1}{2} h_{\nu \sigma}\left(\nabla_{\mu} \zeta^{\sigma}+\nabla_{\sigma} \zeta_{\mu}\right)\right] *\left(d x^{\mu} \wedge d x^{\nu}\right)
\end{gathered}
$$

where $*$ denotes the Hodge dual. Note that the formula also applies to higher dimensions. The Dirac bracket algebra of the charges includes a central term [14]:

$$
\left\{Q_{\zeta_{(m)}}, Q_{\zeta_{(n)}}\right\}_{D B}=Q_{\left[\zeta_{(m)}, \zeta_{(n)}\right]}+\frac{1}{8 \pi} \int_{\partial \Sigma} k_{\zeta_{(m)}}\left[\mathcal{L}_{\zeta_{(n)}} g, g\right]
$$

where $\mathcal{L}_{\zeta} g_{\mu \nu}=\zeta^{\rho} \partial_{\rho} g_{\mu \nu}+g_{\rho \nu} \partial_{\mu} \zeta^{\rho}+g_{\mu \rho} \partial_{\nu} \zeta^{\rho}$ is the Lie derivative of $g_{\mu \nu}$ with respect to $\zeta$. Translated into modes, the corresponding commutator algebra is given by

$$
\left[L_{m}, L_{n}\right]=(m-n) L_{m+n}+\frac{1}{12} c_{L}\left(m^{3}+\alpha m\right) \delta_{m+n, 0}
$$


with the central charge $c_{L}$ given by

$$
\frac{1}{8 \pi} \int_{\partial \Sigma} k_{\zeta_{(m)}}\left[\mathcal{L}_{\zeta_{(n)}} g, g\right]=-\frac{\mathrm{i}}{12} c_{L}\left(m^{3}+\alpha m\right) \delta_{m+n, 0}
$$

(The term linear in $m$ plays no essential rôle here, since it can be shifted arbitrarily by $c$-number shifts of the generators $L_{m}$. Thus the value of the constant $\alpha$ is unimportant.)

Evaluating the integral (2.21) for the case of the four-dimensional near-horizon extremal Kerr-AdS metric (2.13), we find

$$
\begin{aligned}
\frac{1}{8 \pi} \int_{\partial \Sigma} k_{\zeta_{(m)}}\left[\mathcal{L}_{\zeta_{(n)}} g, g\right]= & -\frac{\mathrm{i} m^{3} r_{0}^{2} \sqrt{\left(1-r_{0}^{2} \ell^{-2}\right)\left(1+3 r_{0}^{2} \ell^{-2}\right)}}{1+6 r_{0}^{2} \ell^{-2}-3 r_{0}^{4} \ell^{-4}} \\
& -\frac{2 \mathrm{i} m r_{0}^{2} \sqrt{\left(1-r_{0}^{2} \ell^{-2}\right)\left(1+3 r_{0}^{2} \ell^{-2}\right)}}{\left(1-3 r_{0}^{2} \ell^{-2}\right)^{2}}
\end{aligned}
$$

and hence the central charge is

$$
c_{L}=\frac{12 r_{0}^{2} \sqrt{\left(1-r_{0}^{2} \ell^{-2}\right)\left(1+3 r_{0}^{2} \ell^{-2}\right)}}{1+6 r_{0}^{2} \ell^{-2}-3 r_{0}^{4} \ell^{-4}} .
$$

\subsection{Microscopic entropy and the Cardy formula}

Following [1], one can adopt the Frolov-Thorne vacuum [15] to provide a definition of the vacuum state for the extreme Kerr-AdS metric. Quantum fields for the general (nonextremal) Kerr-AdS metric (2.1) can be expanded in eigenstates with asymptotic energy $\omega$ and angular momentum $m$, with $\hat{t}$ and $\hat{\phi}$ dependence $e^{-\mathrm{i} \omega \hat{t}+\mathrm{i} m \hat{\phi}}$. In terms of the the redefined $t$ and $\phi$ coordinates of the extremal near-horizon limit, given by (2.5) and (2.6), we have

$$
e^{-\mathrm{i} \omega \hat{t}+\mathrm{i} m \hat{\phi}}=e^{-\mathrm{i} n_{R} t+\mathrm{i} n_{L} \phi}
$$

where

$$
n_{L}=m, \quad n_{R}=\frac{\left(r_{0}^{2}+a^{2}\right) \omega-a m \Xi}{\lambda r_{0} V} .
$$

The left-moving and right-moving temperatures $T_{L}$ and $T_{R}$ are then defined by writing the Boltzmann factor $e^{-\left(\omega-m \Omega_{\phi}\right) / T_{H}}$ as

$$
e^{-\left(\omega-m \Omega_{\phi}\right) / T_{H}}=e^{-n_{L} / T_{L}-n_{R} / T_{R}} .
$$

This allows us to read off

$$
T_{L}=\frac{T_{H}}{a \Xi\left(r_{0}^{2}+a^{2}\right)^{-1}-\Omega_{\phi}}, \quad T_{R}=\frac{\left(r_{0}^{2}+a^{2}\right) T_{H}}{\lambda r_{0} V} .
$$


We now take the extremal limit, finding 1

$$
T_{L}=\frac{1+6 r_{0}^{2} \ell^{-2}-3 r_{0}^{4} \ell^{-4}}{2 \pi\left(1-3 r_{0}^{2} \ell^{-2}\right) \sqrt{\left(1+3 r_{0}^{2} \ell^{-2}\right)\left(1-r_{0}^{2} \ell^{-2}\right)}}, \quad T_{R}=0 .
$$

In the case of vanishing cosmological constant, i.e. $\ell^{-1}=0$, the result reduces to $T_{L}=$ $1 /(2 \pi)$, given in $[1]$.

From the Cardy formula for the entropy of a unitary conformal field theory at temperature $T_{L}$, the microscopic entropy is given by

$$
S=\frac{1}{3} \pi^{2} c_{L} T_{L}
$$

From (2.23) and (2.28), we therefore obtain the microscopic entropy

$$
S=\frac{2 \pi r_{0}^{2}}{1-3 r_{0}^{2} \ell^{-2}}
$$

for the CFT dual to the extremal Kerr-AdS black hole. This agrees perfectly with the Bekenstein-Hawking entropy which given in (2.2), after taking the extremal limit.

\section{Five-Dimensional Extremal Rotating Black Holes}

\subsection{The extremal near-horizon metric}

The general result for the metric of a five-dimensional rotating black hole with $S^{3}$ horizon topology, satisfying the Einstein equation $R_{\mu \nu}=-4 \ell^{-2} g_{\mu \nu}$, was obtained by Hawking, Hunter and Taylor-Robinson [11]. This metric, which generalises the Ricci-flat rotating black hole of Myers and Perry [8], is given by

$$
\begin{aligned}
d s^{2}= & -\frac{\Delta}{\rho^{2}}\left(d \hat{t}-\frac{a \sin ^{2} \theta}{\Xi_{a}} d \phi_{1}-\frac{b \cos ^{2} \theta}{\Xi_{b}} d \phi_{2}\right)^{2}+\frac{\Delta_{\theta} \sin ^{2} \theta}{\rho^{2}}\left(a d \hat{t}-\frac{\left(\hat{r}^{2}+a^{2}\right)}{\Xi_{a}} d \phi_{1}\right)^{2} \\
& +\frac{\Delta_{\theta} \cos ^{2} \theta}{\rho^{2}}\left(b d \hat{t}-\frac{\left(\hat{r}^{2}+b^{2}\right)}{\Xi_{b}} d \phi_{2}\right)^{2}+\frac{\rho^{2}}{\Delta} d \hat{r}^{2}+\frac{\rho^{2}}{\Delta \theta} d \theta^{2} \\
& +\frac{1+\hat{r}^{2} \ell^{-2}}{\hat{r}^{2} \rho^{2}}\left(a b d \hat{t}-\frac{b\left(\hat{r}^{2}+a^{2}\right) \sin ^{2} \theta}{\Xi_{a}} d \phi_{1}-\frac{a\left(\hat{r}^{2}+b^{2}\right) \cos ^{2} \theta}{\Xi_{b}} d \phi_{2}\right)^{2},
\end{aligned}
$$

where

$$
\begin{aligned}
& \Delta=\frac{1}{\hat{r}^{2}}\left(\hat{r}^{2}+a^{2}\right)\left(\hat{r}^{2}+b^{2}\right)\left(1+\hat{r}^{2} \ell^{-2}\right)-2 M, \quad \Delta_{\theta}=1-a^{2} \ell^{-2} \cos ^{2} \theta-b^{2} \ell^{-2} \sin ^{2} \theta, \\
& \rho^{2}=\hat{r}^{2}+a^{2} \cos ^{2} \theta+b^{2} \sin ^{2} \theta, \quad \Xi_{a}=1-a^{2} \ell^{-2}, \quad \Xi_{b}=1-b^{2} \ell^{-2} .
\end{aligned}
$$

\footnotetext{
${ }^{1}$ Note that had we chosen to work in coordinate frame in which the angular velocity at infinity were different (such as an asymptotically static frame), then the expressions for $\Omega_{\phi}$ and for the $\hat{t}$-dependent shift in (2.5) would each be be displaced by the same additive constant, and so the final expression for $T_{L}$ in (2.27) would be the same.
} 
Note that in this coordinate system, the metric is asymptotic to $\mathrm{AdS}_{5}$ in a rotating frame, with angular velocities $\Omega_{\phi_{1}}^{\infty}=-a \ell^{-2}$ and $\Omega_{\phi_{2}}^{\infty}=-b \ell^{-2}$.

The Hawking temperature, Bekenstein-Hawking entropy, the angular momenta and the angular velocities on the horizon (in the asymptotically rotating frame) are given by

$$
\begin{aligned}
T_{H} & =\frac{r_{+}^{4}-a^{2} b^{2}+g^{2} r_{+}^{4}\left(2 r_{+}^{2}+a^{2}+b^{2}\right)}{2 \pi r_{+}\left(r_{+}^{2}+a^{2}\right)\left(r_{+}^{2}+b^{2}\right)}, \\
S_{B H} & =\frac{\pi^{2}\left(r_{+}^{2}+a^{2}\right)\left(r_{+}^{2}+b^{2}\right)}{2 r_{+} \Xi_{a} \Xi_{b}} \\
J_{\phi_{1}} & =\frac{\pi M a}{2 \Xi_{1}^{2} \Xi_{2}}, \quad J_{\phi_{2}}=\frac{\pi M b}{2 \Xi_{1} \Xi_{2}^{2}}, \\
\Omega_{\phi_{1}} & =\frac{a \Xi_{a}}{r_{+}^{2}+a^{2}}, \quad \Omega_{\phi_{2}}=\frac{b \Xi_{b}}{r_{+}^{2}+b^{2}} .
\end{aligned}
$$

Extremality occurs if $\Delta$ has a double zero at the horizon radius $r=r_{0}$, implying that the Hawking temperature vanishes. These conditions can conveniently be solved for $M$ and $\ell^{-2}$ :

$$
M=\frac{\left(r_{0}^{2}+a^{2}\right)^{2}\left(r_{0}^{2}+b^{2}\right)^{2}}{2 r_{0}^{4}\left(2 r_{0}^{2}+a^{2}+b^{2}\right)}, \quad \frac{\left(a b-r_{0}^{2}\right)\left(a b+r_{0}^{2}\right)}{r_{0}^{4}\left(2 r_{0}^{2}+a^{2}+b^{2}\right)}=\ell^{-2} .
$$

With these parameters, we have $\Delta=\left(r-r_{0}\right)^{2} V+\mathcal{O}\left(\left(r-r_{0}\right)^{3}\right)$, where

$$
V=4+4\left(3 r_{0}^{2}+a^{2}+b^{2}\right) \ell^{-2}
$$

To extract the near-horizon geometry we first make the coordinate transformations

$$
\begin{array}{ll}
\hat{r}=r_{0}(1+\lambda y), & \phi_{1} \rightarrow \phi_{1}+\alpha_{1} \hat{t}, \quad \phi_{2} \rightarrow \phi_{2}+\alpha_{2} \hat{t}, \\
\alpha_{1}=\frac{a \Xi_{a}}{r_{0}^{2}+a^{2}}, & \alpha_{2}=\frac{b \Xi_{b}}{r_{0}^{2}+b^{2}} .
\end{array}
$$

then scale the time coordinate

$$
\hat{t}=\beta t, \quad \beta=\frac{\left(r_{0}^{2}+a^{2}\right)\left(r_{0}^{2}+b^{2}\right)}{\lambda r_{0}^{3} V},
$$

and finally send $\lambda \rightarrow 0$. The metric becomes

$$
\begin{aligned}
d s^{2}= & \frac{\rho_{0}^{2}}{V}\left(-y^{2} d t+\frac{d y^{2}}{y^{2}}\right)+\frac{\rho_{0}^{2} d \theta^{2}}{\Delta_{\theta}}+\frac{\Delta_{\theta} \sin ^{2} \theta}{\rho_{0}^{2}}\left(\frac{2 a\left(r_{0}^{2}+b^{2}\right)}{r_{0} V} y d t+\frac{r_{0}^{2}+a^{2}}{\Xi_{a}} d \phi_{1}\right)^{2} \\
& +\frac{\Delta_{\theta} \cos ^{2} \theta}{\rho_{0}^{2}}\left(\frac{2 b\left(r_{0}^{2}+a^{2}\right)}{r_{0} V} y d t+\frac{r_{0}^{2}+b^{2}}{\Xi_{b}} d \phi_{2}\right)^{2} \\
& +\frac{1+r_{0}^{2} \ell^{-1}}{r_{0}^{2} \rho_{0}^{2}}\left(\frac{2 a b \rho_{0}^{2}}{r_{0} V} y d t+\frac{b\left(r_{0}^{2}+a^{2}\right) \sin ^{2} \theta}{\Xi_{a}} d \phi_{1}+\frac{a\left(r_{0}^{2}+b^{2}\right) \cos ^{2} \theta}{\Xi_{b}} d \phi_{2}\right)^{2}
\end{aligned}
$$

where

$$
\rho_{0}^{2}=r_{0}^{2}+a^{2} \cos ^{2} \theta+b^{2} \sin ^{2} \theta, \quad \Delta_{\theta}=1-a^{2} \ell^{-2} \cos ^{2} \theta-b^{2} \ell^{-2} \sin ^{2} \theta
$$


Finally, we can transform the $\mathrm{AdS}_{2}$ Poincaré coordinates $y$ and $t$ to global coordinates $r$ and $\tau$ by using the transformations (2.9), and sending

$$
\phi_{1} \longrightarrow \phi_{1}+\frac{2 a \gamma\left(r_{0}^{2}+b^{2}\right) \Xi_{a}}{r_{0} V\left(r_{0}^{2}+a^{2}\right)}, \quad \phi_{2} \longrightarrow \phi_{2}+\frac{2 b \gamma\left(r_{0}^{2}+a^{2}\right) \Xi_{b}}{r_{0} V\left(r_{0}^{2}+b^{2}\right)},
$$

where $\gamma$ is given by (2.11). The five-dimensional near-horizon extremal black hole metric (3.8) becomes

$$
\begin{aligned}
d s^{2}= & \frac{\rho_{0}^{2}}{V}\left(-\left(1+r^{2}\right) d \tau^{2}+\frac{d r^{2}}{1+r^{2}}\right)+\frac{\rho_{0}^{2} d \theta^{2}}{\Delta_{\theta}}+\frac{\Delta_{\theta} \sin ^{2} \theta}{\rho_{0}^{2}}\left(\frac{2 a\left(r_{0}^{2}+b^{2}\right)}{r_{0} V} r d \tau+\frac{r_{0}^{2}+a^{2}}{\Xi_{a}} d \phi_{1}\right)^{2} \\
& +\frac{\Delta_{\theta} \cos ^{2} \theta}{\rho_{0}^{2}}\left(\frac{2 b\left(r_{0}^{2}+a^{2}\right)}{r_{0} V} r d \tau+\frac{r_{0}^{2}+b^{2}}{\Xi_{b}} d \phi_{2}\right)^{2} \\
& +\frac{1+r_{0}^{2} \ell^{-2}}{r_{0}^{2} \rho_{0}^{2}}\left(\frac{2 a b \rho_{0}}{r_{0} V} r d \tau+\frac{b\left(r_{0}^{2}+a^{2}\right) \sin ^{2} \theta}{\Xi_{a}} d \phi_{1}+\frac{a\left(r_{0}^{2}+b^{2}\right) \cos ^{2} \theta}{\Xi_{b}} d \phi_{2}\right)^{2} .
\end{aligned}
$$

(This result also appears, with $\ell^{-1}=0$, in [16], and with $\ell^{-1}>0$ in [17].)

\subsection{Central charge and microscopic entropy}

We can introduce an asymptotic symmetry group for this five-dimensional extremal black hole in a manner that closely parallels the four-dimensional example in [1]. The difference now is that instead of having just a single algebra of reparameterisations of the circle, as in (2.14), there are now two, associated with the two independent azimuthal coordinates $\phi_{1}$ and $\phi_{2}$ :

$$
\begin{aligned}
\zeta_{(n)}^{(1)} & =-e^{-\mathrm{i} n \phi_{1}} \frac{\partial}{\partial \phi_{1}}-\mathrm{i} n r e^{-\mathrm{i} n \phi_{1}} \frac{\partial}{\partial r}, \\
\zeta_{(n)}^{(2)} & =-e^{-\mathrm{i} n \phi_{2}} \frac{\partial}{\partial \phi_{2}}-\mathrm{i} n r e^{-\mathrm{i} n \phi_{2}} \frac{\partial}{\partial r},
\end{aligned}
$$

We can now calculate the central charges for each of the independent (mutually commuting) Virasoro algebras, using the formalism summarised in section 2.2. We find

$$
\begin{aligned}
& c_{\phi_{1}}=\frac{6 \pi a\left(r_{0}^{2}+b^{2}\right)^{2}}{r_{0}^{2} \Xi_{b} V}=\frac{3 \pi a r_{0}^{6}\left(r_{0}^{2}+b^{2}\right)\left(2 r_{0}^{2}+a^{2}+b^{2}\right)^{2}}{2\left(2 r_{0}^{4}+a^{2} r_{0}^{2}-a^{2} b^{2}\right)\left(-r_{0}^{6}+3 a^{2} b^{2} r_{0}^{2}+a^{2} b^{4}+a^{4} b^{2}\right)}, \\
& c_{\phi_{2}}=\frac{6 \pi b\left(r_{0}^{2}+a^{2}\right)^{2}}{r_{0}^{2} \Xi_{a} V}=\frac{3 \pi b r_{0}^{6}\left(r_{0}^{2}+a^{2}\right)\left(2 r_{0}^{2}+a^{2}+b^{2}\right)^{2}}{2\left(2 r_{0}^{4}+b^{2} r_{0}^{2}-a^{2} b^{2}\right)\left(-r_{0}^{6}+3 a^{2} b^{2} r_{0}^{2}+a^{2} b^{4}+a^{4} b^{2}\right)} .
\end{aligned}
$$

There are now three Frolov-Thorne temperatures associated with the quantum field theory of the extremal five-dimensional Kerr-AdS metric, which we shall denote by $T_{t}, T_{\phi_{1}}$ and $T_{\phi_{2}}$. Thus we consider

$$
e^{-\mathrm{i} \omega \hat{t}+\mathrm{i} m_{1} \hat{\phi}_{1}+\mathrm{i} m_{2} \hat{\phi}_{2}}=e^{-\mathrm{i} n_{t} t+\mathrm{i} n_{\phi_{1}} \phi_{1}+\mathrm{i} n_{\phi_{2}} \phi_{2}},
$$


where

$$
\hat{\phi}_{1}=\phi_{1}+\alpha_{1} \hat{t}, \quad \hat{\phi}_{2}=\phi_{2}+\alpha_{2} \hat{t}, \quad \hat{t}=\beta t .
$$

This implies that

$$
n_{\phi_{1}}=m_{1}, \quad n_{\phi_{2}}=m_{2}, \quad \omega=\frac{n_{t}}{\beta}+\alpha_{1} n_{\phi_{1}}+\alpha_{2} n_{\phi_{2}} .
$$

Now we consider

$$
\exp \left(-\frac{\omega-m_{1} \Omega_{\phi_{1}}-m_{2} \Omega_{\phi_{2}}}{T_{H}}\right)=\exp \left(-\frac{n_{t}}{T_{t}}-\frac{n_{\phi_{1}}}{T_{\phi_{1}}}-\frac{n_{\phi_{2}}}{T_{\phi_{2}}}\right)
$$

Thus we have

$$
T_{t}=\beta T, \quad T_{\phi_{1}}=\frac{T_{H}}{\alpha_{1}-\Omega_{1}}, \quad T_{\phi_{2}}=\frac{T_{H}}{\alpha_{2}-\Omega_{2}} .
$$

where $\alpha_{1}, \alpha_{2}$ and $\beta$ are given in (3.6) and (3.7). In the extremal limit, these quantities evaluate to give

$$
\begin{aligned}
T_{t} & =0 \\
T_{\phi_{1}} & =\frac{r_{0}\left(r_{0}^{2}+a^{2}\right) V}{4 \pi a \Xi_{a}\left(r_{0}^{2}+b^{2}\right)}=\frac{r_{0}\left(-r_{0}^{6}+3 a^{2} b^{2} r_{0}^{2}+a^{2} b^{4}+a^{4} b^{2}\right)}{\pi a\left(r_{0}^{2}+b^{2}\right)\left(2 r_{0}^{4}+b^{2} r_{0}^{2}-a^{2} b^{2}\right)} \\
T_{\phi_{2}} & =\frac{r_{0}\left(r_{0}^{2}+b^{2}\right) V}{4 \pi b \Xi_{b}\left(r_{0}^{2}+a^{2}\right)}=\frac{r_{0}\left(-r_{0}^{6}+3 a^{2} b^{2} r_{0}^{2}+a^{2} b^{4}+a^{4} b^{2}\right)}{\pi b\left(r_{0}^{2}+a^{2}\right)\left(2 r_{0}^{4}+a^{2} r_{0}^{2}-a^{2} b^{2}\right)}
\end{aligned}
$$

We can use the Cardy formula to calculate the microscopic entropy of the CFT associated with each of the $\phi_{1}$ and $\phi_{2}$ circles. The central charges (3.13) and Frolov-Thorne temperatures (3.19) are different for the two CFTs, but nevertheless we find that the microscopic entropy of each CFT is the same:

$$
S=\frac{1}{3} \pi^{2} c_{\phi_{1}} T_{\phi_{1}}=\frac{1}{3} \pi^{2} c_{\phi_{2}} T_{\phi_{2}}
$$

Furthermore, this entropy agrees precisely with the Bekenstein-Hawking entropy of the extremal five-dimensional black hole, which is obtained by substituting the extremality conditions (3.4) into (3.3):

$$
S_{B H}=\frac{\pi^{2} r_{0}^{7}\left(2 r_{0}^{2}+a^{2}+b^{2}\right)^{2}}{2\left(2 r_{0}^{4}+a^{2} r_{0}^{2}-a^{2} b^{2}\right)\left(2 r_{0}^{4}+b^{2} r_{0}^{2}-a^{2} b^{2}\right)} .
$$

In the Ricci-flat case $\ell^{-1}=0$, which implies $r_{0}^{2}=a b$, we have the much simpler expressions

$$
\begin{aligned}
& T_{\phi_{1}}=\frac{a}{\pi \sqrt{a b}}, \quad T_{\phi_{2}}=\frac{b}{\pi \sqrt{a b}}, \quad c_{\phi_{1}}=\frac{3}{2} \pi b(a+b)^{2}, \quad c_{\phi_{2}}=\frac{3}{2} \pi a(a+b)^{2}, \\
& S=\frac{1}{2} \pi^{2}(a+b)^{2} \sqrt{a b} .
\end{aligned}
$$




\section{Kerr-AdS/CFT Correspondence in Higher Dimensions}

The general Kerr-AdS metrics in arbitrary higher dimensions were obtained in $[9,10]$. The solutions were later generalised further to include NUT charges [18]. Although we shall not be including the NUT parameters in our calculations, the form of the metrics obtained in [18] will be the most convenient one for our purposes. We shall first review the Kerr-AdS metrics written in this form, and then obtain their extremal limits. It is convenient to consider the cases when the spacetime dimension is odd, $D=2 n+1$, and even, $D=2 n$, separately.

\section{1 $D=2 n+1$ dimensional Kerr-AdS black holes, and extremal limit}

We shall use the coordinates introduced in section 2 of [18], except that here we shall shift the azimuthal angles by adding convenient constant multiples of the time coordinate $\hat{t}$. Although the resulting coordinate frame is asymptotically rotating, it has the advantage of simplifying the expression for the metric. (As we discussed in section 2.3 for the fourdimensional example, the final results are independent of the choice of frame.) The metric is then given by

$$
\begin{aligned}
d s^{2}= & \frac{U}{X} d \hat{r}^{2}+\sum_{\alpha=1}^{n-1} \frac{U_{\alpha}}{X_{\alpha}} d y_{\alpha}^{2}-\frac{X}{U}\left[d \hat{t}-\sum_{i=1}^{n} a_{i}^{2} \gamma_{i} \frac{d \hat{\phi}_{i}}{\epsilon_{i}}\right]^{2} \\
& +\sum_{\alpha=1}^{n-1} \frac{X_{\alpha}}{U_{\alpha}}\left[d \hat{t}-\sum_{i=1}^{n} \frac{a_{i}^{2}\left(\hat{r}^{2}+a_{i}^{2}\right) \gamma_{i}}{a_{i}^{2}-y_{\alpha}^{2}} \frac{d \hat{\phi}_{i}}{\epsilon_{i}}\right]^{2}+\frac{\prod_{k=1}^{n} a_{k}^{2}}{\hat{r}^{2} \prod_{\alpha=1}^{n-1} y_{\alpha}^{2}}\left[d \hat{t}-\sum_{i=1}^{n}\left(\hat{r}^{2}+a_{i}^{2}\right) \gamma_{i} \frac{d \hat{\phi}_{i}}{\epsilon_{i}}\right]^{2},
\end{aligned}
$$

where

$$
\begin{aligned}
U & =\prod_{\alpha=1}^{n-1}\left(\hat{r}^{2}+y_{\alpha}^{2}\right), \quad U_{\alpha}=-\left(\hat{r}^{2}+y_{\alpha}^{2}\right) \prod_{\beta=1}^{\prime n-1}\left(y_{\beta}^{2}-y_{\alpha}^{2}\right), \quad 1 \leq \alpha \leq n-1, \\
\epsilon_{i} & =a_{i} \Xi_{i} \prod_{k=1}^{\prime n}\left(a_{i}^{2}-a_{k}^{2}\right), \quad \gamma_{i}=\prod_{\alpha=1}^{n-1}\left(a_{i}^{2}-y_{\alpha}^{2}\right), \quad 1 \leq i \leq n, \\
X & =\frac{1+\hat{r}^{2} \ell^{-2}}{\hat{r}^{2}} \prod_{k=1}^{n}\left(\hat{r}^{2}+a_{k}^{2}\right)-2 M, \quad \Xi_{i}=1-a_{i}^{2} \ell^{-2}, \\
X_{\alpha} & =\frac{1-y_{\alpha}^{2} \ell^{-2}}{y_{\alpha}^{2}} \prod_{k=1}^{n}\left(a_{k}^{2}-y_{\alpha}^{2}\right)+2 L_{\alpha}, \quad 1 \leq \alpha \leq n-1 .
\end{aligned}
$$

Note that the notation $\Pi^{\prime}$ indicates that the term in the full product that vanishes is to be omitted. We shall focus on the Kerr-AdS solutions, corresponding to setting all the NUT charges zero, i.e. $L_{\alpha}=0$ for all $1 \leq \alpha \leq n-1$. Without loss of generality, we may order the rotation parameters such that $a_{1} \leq a_{2} \leq \cdots \leq a_{n}$, in which case the compact 
coordinates $y_{\alpha}$ must lie in the ranges $a_{\alpha} \leq y_{\alpha} \leq a_{\alpha+1}$. The asymptotic region, where the metric approaches $\mathrm{AdS}_{2 n+1}$ written in global coordinates, is at $\hat{r}=\infty$. The $n$ azimuthal angles $\hat{\phi}_{i}$ each have period $2 \pi$, and the asymptotic angular velocities are $\Omega_{i}^{\infty}=-a_{i} \ell^{-2}$.

The horizon is located at $\hat{r}=r_{+}$, where $r_{+}$is the largest root of the polynomial function $X$. The thermodynamic quantities for the Kerr-AdS solutions were obtained in [19]. The mass, angular momenta and Bekenstein-Hawking entropy are given by

$$
E=\frac{m \mathcal{A}_{D-2}}{4 \pi\left(\prod_{j} \Xi_{j}\right)}\left(\sum_{i=1}^{n} \frac{1}{\Xi_{i}}-\frac{1}{2}\right), \quad J_{i}=\frac{M a_{i} \mathcal{A}_{D-2}}{4 \pi \Xi_{i}\left(\prod_{j} \Xi_{j}\right)}, \quad S_{B H}=\frac{\mathcal{A}_{D-2}}{4 \pi r_{+}} \prod_{i=1}^{n} \frac{r_{+}^{2}+a_{i}^{2}}{\Xi_{i}},
$$

where $\mathcal{A}_{D-2}=2 \pi^{(D-1) / 2} / \Gamma[(D-1) / 2]$ is the volume of the unit $(D-2)$-sphere. The Hawking temperature and the angular velocities on the horizon (measured in the asymptotically rotating frame) are given by

$$
\begin{aligned}
T_{H} & =\frac{1}{2 \pi}\left[r_{+}\left(1+r_{+}^{2} \ell^{-2}\right) \sum_{i} \frac{1}{r_{+}^{2}+a_{i}^{2}}-\frac{1}{r_{+}}\right], \\
\Omega_{i} & =\frac{a_{i} \Xi_{i}}{r_{+}^{2}+a_{i}^{2}} .
\end{aligned}
$$

We now consider the extremal limit. It occurs when the function $X$ has a double zero, implying that the parameters must be chosen so that

$$
\left.X\right|_{\hat{r}=r_{0}}=0,\left.\quad X^{\prime}\right|_{\hat{r}=r_{0}}=0,
$$

where $\hat{r}=r_{0}$ is the horizon of the extremal black hole. Near the horizon, the function $X$ can be expanded as

$$
X=\left(\hat{r}-r_{0}\right)^{2} V+\mathcal{O}\left(\left(\hat{r}-r_{0}\right)^{3}\right)
$$

where

$$
V=\left.\frac{1}{2} X^{\prime \prime}\right|_{\hat{r}=r_{0}}
$$

To extract the near-horizon geometry, we first make the coordinate redefinition

$$
\hat{r}=r_{0}(1+\lambda y), \quad \hat{\phi}_{i}=\phi_{i}+\alpha_{i} \hat{t}, \quad \alpha_{i}=\frac{a_{i} \Xi_{i}}{r_{0}^{2}+a_{i}^{2}},
$$

and then make a scaling of the time coordinate

$$
\hat{t}=\beta t, \quad \beta=\frac{\prod_{i}\left(r_{0}^{2}+a_{i}^{2}\right)}{\lambda r_{0}^{3} V} .
$$


Finally, taking the limit $\lambda \rightarrow 0$ we obtain the near-horizon geometry

$$
\begin{aligned}
d s^{2}= & \frac{\widetilde{U}}{V}\left(-y^{2} d t^{2}+\frac{d y^{2}}{y^{2}}\right)+\sum_{\alpha=1}^{n-1} \frac{\widetilde{U}_{\alpha}}{X_{\alpha}} d y_{\alpha}^{2} \\
& +\sum_{\alpha=1}^{n-1} \frac{X_{\alpha}}{\widetilde{U}_{\alpha}}\left[\frac{2 r_{0} \prod_{\beta}\left(r_{0}^{2}+y_{\beta}^{2}\right)}{V\left(r_{0}^{2}+y_{\alpha}^{2}\right)} y d t+\sum_{i=1}^{n} \frac{a_{i}^{2}\left(r_{0}^{2}+a_{i}^{2}\right) \gamma_{i}}{a_{i}^{2}-y_{\alpha}^{2}} \frac{d \phi_{i}}{\epsilon_{i}}\right]^{2} \\
& +\frac{\prod_{k=1}^{n} a_{k}^{2}}{r_{0}^{2} \prod_{\alpha=1}^{n-1} y_{\alpha}^{2}}\left[\frac{2 \prod_{\beta}\left(r_{0}^{2}+y_{\beta}^{2}\right)}{r_{0} V} y d t+\sum_{i=1}^{n}\left(r_{0}^{2}+a_{i}^{2}\right) \gamma_{i} \frac{d \phi_{i}}{\epsilon_{i}}\right]^{2},
\end{aligned}
$$

where $\widetilde{U}$ and $\widetilde{U}_{\alpha}$ are $U$ and $U_{\alpha}$ given in (4.2), but with $\hat{r}$ replaced by $r_{0}$.

As in the four-dimensional and five-dimensional examples we discussed in previous sections, we can finally transform from Poincaré coordinates $t$ and $y$ to global coordinates $\tau$ and $r$, by means of the redefinitions (2.9), together with appropriate shifts of the azimuthal angles, giving the near-horizon metric in the form

$$
\begin{aligned}
d s^{2}= & \frac{\widetilde{U}}{V}\left(-\left(1+r^{2}\right) d \tau^{2}+\frac{d r^{2}}{1+r^{2}}\right)+\sum_{\alpha=1}^{n-1} \frac{\widetilde{U}_{\alpha}}{X_{\alpha}} d y_{\alpha}^{2} \\
& +\sum_{\alpha=1}^{n-1} \frac{X_{\alpha}}{\widetilde{U}_{\alpha}}\left[\frac{2 r_{0} \prod_{\beta}\left(r_{0}^{2}+y_{\beta}^{2}\right)}{V\left(r_{0}^{2}+y_{\alpha}^{2}\right)} r d \tau+\sum_{i=1}^{n} \frac{a_{i}^{2}\left(r_{0}^{2}+a_{i}^{2}\right) \gamma_{i}}{a_{i}^{2}-y_{\alpha}^{2}} \frac{d \phi_{i}}{\epsilon_{i}}\right]^{2} \\
& +\frac{\prod_{k=1}^{n} a_{k}^{2}}{r_{0}^{2} \prod_{\alpha=1}^{n-1} y_{\alpha}^{2}}\left[\frac{2 \prod_{\beta}\left(r_{0}^{2}+y_{\beta}^{2}\right)}{r_{0} V} r d \tau+\sum_{i=1}^{n}\left(r_{0}^{2}+a_{i}^{2}\right) \gamma_{i} \frac{d \phi_{i}}{\epsilon_{i}}\right]^{2} .
\end{aligned}
$$

\section{2 $D=2 n$ dimensional Kerr-AdS black holes and extremal limit}

Again we use the same coordinates as those in section 2 of [18], but with shifted azimuthal coordinates chosen so that the metric is simpler, albeit asymptotically rotating. It is given by

$$
\begin{aligned}
d s^{2}= & \frac{U}{X} d \hat{r}^{2}+\sum_{\alpha=1}^{n-1} \frac{U_{\alpha}}{X_{\alpha}} d y_{\alpha}^{2}-\frac{X}{U}\left[d \hat{t}-\sum_{i=1}^{n-1} \frac{\gamma_{i}}{\epsilon_{i}} d \hat{\phi}_{i}\right]^{2} \\
& +\sum_{\alpha=1}^{n-1} \frac{X_{\alpha}}{U_{\alpha}}\left[d \hat{t}-\sum_{i=1}^{n-1} \frac{\left(\hat{r}^{2}+a_{i}^{2}\right) \gamma_{i}}{a_{i}^{2}-y_{\alpha}^{2}} \frac{d \hat{\phi}_{i}}{\epsilon_{i}}\right]^{2}
\end{aligned}
$$


where

$$
\begin{aligned}
U & =\prod_{\alpha=1}^{n-1}\left(\hat{r}^{2}+y_{\alpha}^{2}\right), \quad U_{\alpha}=-\left(\hat{r}^{2}+y_{\alpha}^{2}\right) \prod_{\beta=1}^{\prime n-1}\left(y_{\beta}^{2}-y_{\alpha}^{2}\right), \quad 1 \leq \alpha \leq n-1, \\
\epsilon_{i} & =a_{i} \Xi_{i} \prod_{k=1}^{\prime n-1}\left(a_{i}^{2}-a_{k}^{2}\right), \quad \gamma_{i}=\prod_{\alpha=1}^{n-1}\left(a_{i}^{2}-y_{\alpha}^{2}\right), \quad 1 \leq i \leq n-1 \\
X & =\left(1+\hat{r}^{2} \ell^{-2}\right) \prod_{k=1}^{n-1}\left(\hat{r}^{2}+a_{k}^{2}\right)-2 M \hat{r}, \quad \Xi_{i}=1-a_{i}^{2} \ell^{-2} \\
X_{\alpha} & =-\left(1-y_{\alpha}^{2} \ell^{-2}\right) \prod_{k=1}^{n-1}\left(a_{k}^{2}-y_{\alpha}^{2}\right)+2 L_{\alpha} y_{\alpha} .
\end{aligned}
$$

Turning off the NUT parameters, i.e. $L_{\alpha}=0$, the solution describes the Kerr-AdS black hole in even dimensions $D=2 n$. The coordinate ranges for $y_{\alpha}$ are somewhat different from the odd dimensional case. Without loss of generality, we may order the rotation parameters such that $a_{1} \leq a_{2} \leq \cdots \leq a_{n-1}$, then we have $-a_{1} \leq y_{1} \leq a_{1}$, and $a_{\alpha-1} \leq y_{\alpha} \leq a_{\alpha}$ for $\alpha=2,3, \ldots,(n-1)$. The $(n-1)$ azimuthal angles $\hat{\phi}_{i}$ each have period $2 \pi$. The asymptotic region is at $\hat{r} \rightarrow \infty$, where the metric approaches $\mathrm{AdS}_{2 n}$ in rotating global coordinates, with $\Omega_{i}^{\infty}=-a_{i} \ell^{-2}$. The horizon is at $\hat{r}=r_{+}$, where $r_{+}$is the largest root of the polynomial function $X$. The thermodynamic quantities are given by

$$
\begin{aligned}
T_{H} & =\frac{1}{2 \pi}\left[r_{+}\left(1+r_{+}^{2} \ell^{-2}\right) \sum_{i} \frac{1}{r_{+}^{2}+a_{i}^{2}}-\frac{1-r_{+}^{2} \ell^{-2}}{2 r_{+}}\right], \\
E & =\frac{\mathcal{A}_{D-2} M}{4 \pi\left(\prod_{j} \Xi_{j}\right)} \sum_{i=1}^{n-1} \frac{1}{\Xi_{i}}, \quad S_{B H}=\frac{1}{4} \mathcal{A}_{D-2} \prod_{i=1}^{n-1} \frac{r_{+}^{2}+a_{i}^{2}}{\Xi_{i}} \\
J_{i} & =\frac{a_{i} \mathcal{A}_{D-2} M}{4 \pi \Xi_{i}\left(\prod_{j} \Xi_{j}\right)}, \quad \Omega_{i}=\frac{a_{i} \Xi_{i}}{r_{+}^{2}+a_{i}^{2}},
\end{aligned}
$$

where, as in the previous cases, $\Omega_{i}$ gives the angular velocities on the horizon, in the asymptotically-rotating frame.

As in the case of odd dimensions, the extremal limit is achieved by choosing parameters such that

$$
\left.X\right|_{\hat{r}=r_{0}}=0,\left.\quad X^{\prime}\right|_{\hat{r}=r_{0}}=0 .
$$

The horizon is at $\hat{r}=r_{0}$, and near the horizon, the function $X$ has the expansion $X=$ $\left(\hat{r}-r_{0}\right)^{2} V+\mathcal{O}\left(\left(\hat{r}-r_{0}\right)^{3}\right)$, with $V=\left.\frac{1}{2} X^{\prime \prime}\right|_{\hat{r}=r_{0}}$.

The near-horizon geometry can be extracted by first making the coordinate transformation

$$
\hat{r}=r_{0}(1+\lambda y), \quad \hat{\phi}_{i}=\phi_{i}+\alpha_{i} \hat{t}, \quad \alpha_{i}=\frac{a_{i} \Xi_{i}}{r_{0}^{2}+a_{i}^{2}}
$$


and the time rescaling

$$
\hat{t}=\beta t, \quad \beta=\frac{\prod_{i}^{n-1}\left(r_{0}^{2}+a_{i}^{2}\right)}{\lambda r_{0} V} .
$$

Finally, taking the limit $\lambda \rightarrow 0$ we obtain the near-horizon geometry

$$
\begin{aligned}
d s^{2}= & \frac{\widetilde{U}}{V}\left(-y^{2} d t^{2}+\frac{d y^{2}}{y^{2}}\right)+\sum_{\alpha=1}^{n-1} \frac{\widetilde{U}_{\alpha}}{X_{\alpha}} d y_{\alpha}^{2} \\
& +\sum_{\alpha=1}^{n-1} \frac{X_{\alpha}}{\widetilde{U}_{\alpha}}\left[\frac{2 r_{0} \prod_{\beta}\left(r_{0}^{2}+y_{\beta}^{2}\right)}{V\left(r_{0}^{2}+y_{\alpha}^{2}\right)} y d t+\sum_{i=1}^{n-1} \frac{\left(r_{0}^{2}+a_{i}^{2}\right) \gamma_{i}}{a_{i}^{2}-y_{\alpha}^{2}} \frac{d \phi_{i}}{\epsilon_{i}}\right]^{2},
\end{aligned}
$$

where $\widetilde{U}$ and $\widetilde{U}_{\alpha}$ are $U$ and $U_{\alpha}$ as given in (4.13), but with $\hat{r}$ replaced by $r_{0}$.

In terms of global coordinates on the $\mathrm{AdS}_{2}$, the near-horizon metric becomes

$$
\begin{aligned}
d s^{2}= & \frac{\widetilde{U}}{V}\left(-\left(1+r^{2}\right) d \tau^{2}+\frac{d r^{2}}{1+r^{2}}\right)+\sum_{\alpha=1}^{n-1} \frac{\widetilde{U}_{\alpha}}{X_{\alpha}} d y_{\alpha}^{2} \\
& +\sum_{\alpha=1}^{n-1} \frac{X_{\alpha}}{\widetilde{U}_{\alpha}}\left[\frac{2 r_{0} \prod_{\beta}\left(r_{0}^{2}+y_{\beta}^{2}\right)}{V\left(r_{0}^{2}+y_{\alpha}^{2}\right)} r d \tau+\sum_{i=1}^{n-1} \frac{\left(r_{0}^{2}+a_{i}^{2}\right) \gamma_{i}}{a_{i}^{2}-y_{\alpha}^{2}} \frac{d \phi_{i}}{\epsilon_{i}}\right]^{2} .
\end{aligned}
$$

(Results for the near-horizon geometries for Myers-Perry black holes (i.e. with $\ell^{-1}=0$ ) appear in [20].)

\subsection{Frolov-Thorne vacuum temperature}

There are $[(D+1) / 2]$ Frolov-Thorne temperatures associated with the quantum field theory of the extremal $D$ dimensional metric. We shall denote these by $T_{0}$ and $T_{i}$, with $1 \leq i \leq n$ or $1 \leq i \leq n-1$ when $D=2 n+1$ or $D=2 n$ respectively. Thus we consider

$$
e^{-\mathrm{i} \omega \hat{t}+\mathrm{i} \sum_{i} m_{i} \hat{\phi}_{i}}=e^{-\mathrm{i} n_{0} t+\mathrm{i} \sum_{i} n_{i} \phi_{i}}
$$

where $\left(\hat{t}, \hat{\phi}_{i}\right)$ and $\left(t, \phi_{i}\right)$ are related by (4.8) and (4.9), for both even or odd dimensions. This implies that

$$
n_{i}=m_{i}, \quad \omega=\frac{n_{0}}{\beta}+\sum_{i} \alpha_{i} n_{i}
$$

Now consider

$$
\exp \left(-\frac{\omega-\sum_{i} m_{i} \Omega_{i}}{T_{H}}\right)=\exp \left(-\frac{n_{0}}{T_{0}}-\sum_{i} \frac{n_{i}}{T_{i}}\right)
$$

Thus we have

$$
T_{0}=\beta T_{H}, \quad T_{i}=\frac{T_{H}}{\alpha_{i}-\Omega_{i}} .
$$

Note that the forms for $\alpha_{i}$ and $\Omega_{i}$ are the same for even and odd dimensions. In the extremal limit, it is clear that $T_{0}=0$. For the $T_{i}$, both the numerator and denominator 
vanish, leaving a finite and non-vanishing ratio, and hence we find

$$
T_{i}=\left.\frac{\left(r_{0}^{2}+a_{i}^{2}\right)^{2}}{2 r_{0} a_{i} \Xi_{i}}\left(\frac{\partial T_{H}}{\partial r_{+}}\right)\right|_{r_{+}=r_{0}}=\frac{V r_{0}^{2 \epsilon}\left(r_{0}^{2}+a_{i}^{2}\right)}{4 \pi a_{i} \Xi_{i} r_{0} \prod_{j \neq i}\left(r_{0}^{2}+a_{j}^{2}\right)},
$$

where $\epsilon=0$ and 1 for even and odd dimensions respectively.

\subsection{Central charge and microscopic entropy}

We may again consider a class of diffeomorphisms analogous to (2.14), which give rise to an asymptotic symmetry algebra of transformations obeying boundary conditions of the type discussed in [1]. In the general cases of rotating AdS black holes in $D=2 n+1$ or $D=2 n$ dimensions we shall have $n$ or $(n-1)$ commuting copies of the Virasoro algebra respectively, generated by the diffeomorphisms

$$
\zeta_{(n)}^{i}=-e^{-\mathrm{i} n \phi_{i}} \frac{\partial}{\partial \phi_{i}}-\mathrm{i} n r e^{-\mathrm{i} n \phi_{i}} \frac{\partial}{\partial r} .
$$

The evaluation of the central terms $c_{\phi_{i}}$ in the Virasoro algebras at the level of the Dirac brackets of the charges (2.17), involving the calculation of the surface integral in (2.19) using the extension of (2.18) to higher dimensions, is rather complicated and difficult to perform in a general higher dimension.

The test of the validity of the Kerr/CFT correspondence in the higher dimensions would amount to verifying that the central charges $c_{\phi_{i}}$ are such that

$$
S_{B H}=\frac{1}{3} \pi^{2} c_{\phi_{i}} T_{i} \quad \text { for each } i
$$

where $S_{B H}$ is the Bekenstein-Hawking entropy of the extremal rotating AdS black hole, given by (4.3) or (4.14), with $r_{+}=r_{0}$ subject to $X\left(r_{0}\right)=0$ and $X^{\prime}\left(r_{0}\right)=0$, in odd or even dimensions respectively, and with $T_{i}$ being the Frolov-Thorne vacuum temperature (4.26) for the $i$ 'th Virasoro algebra.

We have calculated the central charges $c_{\phi_{i}}$ explicitly in the cases of $D=6$ and $D=7$ dimensions, and verified that the relations (4.28) are indeed satisfied. Thus in total we have explicit confirmation of the equality of the microscopic entropy calculations and the Bekenstein-Hawking entropy for extremal rotating black holes in dimensions 4, 5, 6 and 7 .

\section{Conclusions}

In this paper, we have extended the recent results in [1] on the Kerr/CFT correspondence for four-dimensional Kerr black holes, by including a cosmological constant and also by 
considering the equivalent correspondence in all higher dimensions. The key observation in [1] was that the asymptotic algebra of a class of diffeomorphisms of the near-horizon geometry of the extremal Kerr black hole, subject to certain boundary conditions, contains a Virasoro algebra related to reparameterisations of the azimuthal coordinate $\phi$ in the Kerr metric. This algebra could be associated with a chiral CFT, and, realised at the level of Dirac brackets of diffeomorphism charges, it has a central term related to the angular momentum of the black hole. It was shown in [1] that the microscopic entropy of the CFT, calculated using the Cardy formula, coincides with the Bekenstein-Hawking entropy of the extremal Kerr black hole.

In dimensions higher than 4, the generalisations of the Kerr and Kerr-AdS metrics have multiple independent angular momenta, associated with rotations in mutually-orthogonal spatial 2-planes [8-11]. This leads to an asymptotic symmetry that includes multiple mutually commuting copies of the Virasoro algebra. The central terms in these Virasoro algebras are all different (when the rotation parameters are unequal), but nevertheless, when we evaluate the microscopic entropy for each of the associated chiral CFTs using the Cardy formula, we find that it agrees precisely with the Bekenstein-Hawking entropy of the extremal Kerr-AdS black hole.

It is intriguing to note that in the case of rotating black holes with a (negative) cosmological constant, there are two ostensibly very different types of duality that can be considered. For asymptotically AdS black holes, one expects that the Bekenstein-Hawking entropy can be calculated in the boundary field theory via the AdS/CFT correspondence [21-23]. In particular, in five dimensions one can consider the duality of the Kerr- $\mathrm{AdS}_{5}$ black hole to a four-dimensional rotating boundary field theory [11]. (In the case of supersymmetric charged rotating black holes in five dimensions [24,25], a boundary free-fermion approximation was used to obtain the entropy up to a numerical factor of order unity [26].) On the other hand, as we have discussed in this paper, there is a Kerr/CFT correspondence in the near-horizon region, in the case of extremal black holes. It would be interesting to see whether this multiplicity of dualities persists in a more general context.

\section{Acknowledgements}

Research supported in part by DOE grant DE-FG03-95ER40917. We are grateful to David Chow for useful discussions. 


\section{References}

[1] M. Guica, T. Hartman, W. Song and A. Strominger, The Kerr/CFT correspondence, arXiv:0809.4266 [hep-th].

[2] S. Carlip, Black hole entropy from conformal field theory in any dimension, Phys. Rev. Lett. 82 (1999) 2828, hep-th/9812013.

[3] S. N. Solodukhin, Conformal description of horizon's states, Phys. Lett. B454 (1999) 213, hep-th/9812056.

[4] M.I. Park, Hamiltonian dynamics of bounded spacetime and black hole entropy: Canonical method, Nucl. Phys. B634 (2002) 339, hep-th/0111224.

[5] G. Kang, J.I. Koga and M.I. Park, Near-horizon conformal symmetry and black hole entropy in any dimension, Phys. Rev. D70 (2004) 024005, hep-th/0402113.

[6] K. Hotta, Y. Hyakutake, T. Kubota, T. Nishinaka and H. Tanida, The CFTinterpolating black hole in three dimensions, arXiv:0811.0910 [hep-th].

[7] B. Carter, Hamilton-Jacobi and Schrödinger separable solutions of Einstein's equations, Commun. Math. Phys. 10, 280 (1968).

[8] R.C. Myers and M.J. Perry, Black holes in higher dimensional space-times, Annals Phys. 172, 304 (1986).

[9] G.W. Gibbons, H. Lü, D.N. Page and C.N. Pope, The general Kerr-de Sitter metrics in all dimensions, J. Geom. Phys. 53, 49 (2005) arXiv:hep-th/0404008.

[10] G.W. Gibbons, H. Lü, D.N. Page and C.N. Pope, Rotating black holes in higher dimensions with a cosmological constant, Phys. Rev. Lett. 93, 171102 (2004) arXiv:hep-th/0409155.

[11] S.W. Hawking, C.J. Hunter and M. Taylor-Robinson, Rotation and the AdS/CFT correspondence, Phys. Rev. D59, 064005 (1999) arXiv:hep-th/9811056].

[12] J.M. Bardeen and G.T. Horowitz, The extreme Kerr throat geometry: A vacuum analog of $A d S_{2} \times S^{2}$ Phys. Rev. D60, 104030 (1999) arXiv:hep-th/9905099].

[13] J.D. Brown and M. Henneaux, Central charges in the canonical realization of asymptotic symmetries: An example from three-dimensional gravity, Commun. Math. Phys. 104, 207 (1986). 
[14] G. Barnich and F. Brandt, Covariant theory of asymptotic symmetries, conservation laws and central charges, Nucl. Phys. B633, 3 (2002) arXiv:hep-th/0111246.

[15] V.P. Frolov and K.S. Thorne, Renormalized stress-energy tensor near the horizon of a slowly evolving, rotating black hole, Phys. Rev. D39 (1989) 2125.

[16] H.K. Kunduri, J. Lucietti and H.S. Reall, Near-horizon symmetries of extremal black holes, Class. Quant. Grav. 24 (2007) 4169, arXiv:0705.4214 [hep-th]].

[17] H.K. Kunduri and J. Lucietti, A classification of near-horizon geometries of extremal vacuum black holes, arXiv:0806.2051 [hep-th].

[18] W. Chen, H. Lü and C.N. Pope, General Kerr-NUT-AdS metrics in all dimensions, Class. Quant. Grav. 23, 5323 (2006) arXiv:hep-th/0604125.

[19] G.W. Gibbons, M.J. Perry and C.N. Pope, The first law of thermodynamics for Kerr anti-de Sitter black holes, Class. Quant. Grav. 22, 1503 (2005) arXiv:hep-th/0408217.

[20] P. Figueras, H.K. Kunduri, J. Lucietti and M. Rangamani, Extremal vacuum black holes in higher dimensions, Phys. Rev. D78 (2008) 044042 [arXiv:0803.2998 [hep-th]].

[21] J.M. Maldacena, The large $N$ limit of superconformal field theories and supergravity, Adv. Theor. Math. Phys. 2, 231 (1998), hep-th/9711200.

[22] S.S. Gubser, I.R. Klebanov and A.M. Polyakov, Gauge theory correlators from noncritical string theory, Phys. Lett. B428, 105 (1998), hep-th/9802109.

[23] E. Witten, Anti-de Sitter space and holography, Adv. Theor. Math. Phys. 2, 253 (1998), hep-th/9802150.

[24] J.B. Gutowski and H.S. Reall, Supersymmetric $A d S_{5}$ black holes, JHEP 0402, 006 (2004) arXiv:hep-th/0401042.

[25] Z.W. Chong, M. Cvetič, H. Lü and C.N. Pope, General non-extremal rotating black holes in minimal five-dimensional gauged supergravity, Phys. Rev. Lett. 95, 161301 (2005) arXiv:hep-th/0506029.

[26] J. Kinney, J.M. Maldacena, S. Minwalla and S. Raju, An index for 4 dimensional super conformal theories, Commun. Math. Phys. 275, 209 (2007) arXiv:hep-th/0510251. 